\title{
$-O$ \\ Capital-Energy Substitution and Shifts in Factor Demand: A Meta-Analysis
}

\author{
Mark J. Koetse \\ Henri L.F. de Groota,b \\ Raymond J.G.M. Floraxa,c
}

\footnotetext{
a Vrije Universiteit Amsterdam,

b Tinbergen Institute,

c Purdue University.
} 


\section{Tinbergen Institute}

The Tinbergen Institute is the institute for economic research of the Erasmus Universiteit Rotterdam, Universiteit van Amsterdam, and Vrije Universiteit Amsterdam.

Tinbergen Institute Amsterdam

Roetersstraat 31

1018 WB Amsterdam

The Netherlands

Tel.: $\quad+31(0) 205513500$

Fax: $\quad+31(0) 205513555$

Tinbergen Institute Rotterdam

Burg. Oudlaan 50

3062 PA Rotterdam

The Netherlands

Tel.: $\quad+31(0) 104088900$

Fax: $\quad+31(0) 104089031$

Most TI discussion papers can be downloaded at http:/ /www.tinbergen.nl. 


\title{
Capital-Energy Substitution and Shifts in Factor Demand: A Meta-Analysis
}

\author{
Mark J. Koetse ${ }^{\mathrm{a}, *}$, Henri L.F. de Groot ${ }^{\mathrm{a}, \mathrm{b}}$, Raymond J.G.M. Florax ${ }^{\mathrm{a}, \mathrm{c}}$ \\ ${ }^{a}$ Department of Spatial Economics, Vrije Universiteit Amsterdam, The Netherlands \\ ${ }^{\mathrm{b}}$ Tinbergen Institute, Amsterdam, The Netherlands \\ ${ }^{\mathrm{c}}$ Department of Agricultural Economics, Purdue University, West Lafayette, United States
}

\begin{abstract}
This paper presents results of a meta-regression analysis on empirical estimates of capital-energy substitution. Theoretically it is clear that a distinction should be made between Morishima substitution elasticities and cross-price elasticities. The former represent purely technical substitution possibilities while the latter include an income effect and therefore represent economic substitution potential. We estimate a meta-regression model with separate coefficients for the two elasticity samples. Our findings suggest that primary model assumptions on returns to scale, technological change and separability of input factors matter for the outcome of a primary study. Aggregation of variables and the type of data used in empirical research are also relevant sources of systematic effect-size variation. Taking these factors into consideration, we compute ideal-typical elasticities for the short, medium and long run. The resulting figures clearly show that substitution elasticities are substantially higher than cross price elasticities. Therefore, despite considerable technical opportunities for capital-energy substitution, they are almost entirely outweighed by the negative income effect brought about by energy price increases; the short and medium run cross price elasticities are not statistically different from zero. In the long run this pattern does not hold. Our findings therefore suggest that actual changes in the demand for capital due to energy price increases take time.
\end{abstract}

Keywords: production function; capital-energy substitution; cross-price elasticity; Morishima substitution elasticity; meta-analysis

JEL-codes: C10; D24; D33; E23; O33; Q40

\footnotetext{
* Corresponding author: Department of Spatial Economics, Vrije Universiteit Amsterdam, De Boelelaan 1105, 1081 HV, Amsterdam, The Netherlands. Tel: + 31 (0)20 598 6168, Fax: + 31 (0)20 598 6004, E-mail: mkoetse@feweb.vu.nl.
} 


\section{Background}

The two oil crises in the 1970s and a growing environmental awareness of societies and governments have induced a steady growth in the availability and performance of energy-saving technologies. The uptake of these technologies is an important means to mitigate greenhouse gas emissions and the depletion of fossil fuels. Moreover, it may reduce an economy's sensitivity to energy-price fluctuations and increase overall efficiency of production. Within the context of production functions, the adoption of an energy-saving technology can be represented by a substitution of capital for energy. Empirical studies differ widely in their substitution estimates, and ultimately provide different answers to the question whether capital and energy are substitutable in a production process, and if so, to what extent. The principal aim of this paper is to investigate the existing heterogeneity in study outcomes, and to provide a more robust estimate of the elasticity of substitution.

The original concept for measuring substitution is the elasticity of substitution, developed by Hicks in 1932. In his words "the elasticity of substitution is a measure of the ease with which the varying factor can be substituted for others" (Hicks, 1932). ${ }^{1}$ Further elaboration reveals that a substitution elasticity between two input factors involves changes in relative factor demand due to changes in relative factor prices, and as such is a two-price-two-factor measure. The economic and econometric evidence supporting capital-energy substitutability is in line with engineering analyses of energyconservation potential. Early studies have shown that average realised energy savings of existing plant and equipment are only a small fraction of the maximum possible energy-saving potential (see Berndt, 1978, for an overview). In contrast, many studies suggest that capital and energy are complements in production, implying that energy-saving potential is purely an engineering, not an economic potential (see also Section 3). Given the fact that the elasticity of substitution between capital and energy is a key parameter in energy demand models and general equilibrium models, and it is highly relevant for various policy questions related to the management of demand and supply of energy, understanding the variation in empirical substitution estimates is crucial. We therefore analyse the available empirical evidence by means of meta-analysis. By using a statistically well-defined framework, this method allows us to quantitatively analyse and synthesise the heterogeneous empirical insights. Although metaanalysis may not be able to provide decisive insights on the empirical correctness of certain theories, assumptions and model specifications, it may contribute to understanding which factors are relevant in explaining the variation in empirical estimates of substitution potential.

The remainder of this paper is organised as follows. In the next section we discuss the translog production function that is used in most primary studies on substitution potential. The parameter estimates provided by estimation of this function are used to derive several elasticity measures. These

\footnotetext{
${ }^{1}$ Reprinted in Hicks (1966, p. 117).
} 
measures are discussed in Section 3. Section 4 describes the study and sample characteristics, while Section 5 discusses the operationalisation of potential sources of systematic effect-size variation. Section 6 continues with the full blown meta-analysis. By estimating meta-models for several elasticity measures, a clear picture arises on the sources of variation, allowing us to assess whether energy and capital can be considered complements or substitutes. Ultimately, clear inferences can be drawn on the potential consequences of different specifications of the production function in primary studies, on separability of input factors, and on the differences between short-run and long-run elasticities. Finally, Section 7 rounds off with a discussion.

\section{Production and cost functions in empirical research}

Empirical studies on capital-energy substitution either apply a nested CES production function or they use a flexible production framework. ${ }^{2}$ The nested CES function is rather restrictive, because when energy and capital are not together in a separate nest, no pure substitution estimate between capital and energy can be derived. Moreover, when capital and energy are together in a separate nest, capital and labour are by construction assumed to be separable in production. This is a rather unrealistic assumption. Therefore, this paper focuses on empirical studies that apply a flexible production framework. Such a framework makes no unnecessary restrictive assumptions on the flexibility of the estimated substitution elasticities and on the optimal path of factor adjustments in reaction to factor price changes.

There are several flexible production functions, but the most popular specification, mainly because it is relatively easy to estimate, is the translog production function introduced by Christensen et al. (1973). ${ }^{3}$ A general problem in estimating a production function is that input factors are likely to be endogenous, thereby violating a basic necessary condition for ordinary least squares to be unbiased. Because factor prices in a cost function most likely do not pose this particular problem, most empirical studies apply the duality theory of production and costs to estimate a translog cost function instead of a translog production function. An extensive discussion of the characteristics and specification of the translog cost function and the derivation of the system of cost-minimising cost-share equations is given in Koetse (2006, pp. 35-40). In this paper a concise discussion of the system estimated in empirical production studies suffices. The cost-minimising cost shares for a capital, labour, energy and materials $(K, L, E, M)$ translog cost function are given by:

\footnotetext{
${ }^{2}$ Studies on capital-energy substitution applying a nested CES function are Prywes (1986), Chang (1994), Khan (1994), Kemfert (1998), Kemfert and Welsch (2000) and Kuper and van Soest (2002).

${ }^{3}$ Examples of other flexible functional forms are the Generalised Leontief function (Diewert, 1971) and the Generalised Cobb Douglas function (Diewert, 1973).
} 


$$
s_{i}=\frac{\partial \ln c(p)}{\partial \ln p_{i}}=\beta_{i}+\sum_{j} \beta_{i j} \ln \left(p_{j}\right)
$$

for $i, j \in\{K, L, E, M\}$. In this equation, $s_{i}$ represent the cost-minimising cost shares, $p_{i}$ and $p_{j}$ factor prices, and $c(p)$ total production costs which are dependent on factor prices $p$. The parameters to be estimated by the model are the $\beta_{i}$ and $\beta_{i j}$, where the former are referred to as the distribution parameters and the latter represent Slutsky substitution elasticities. Since Slutsky elasticities are symmetric by definition, we have to impose:

$$
\beta_{i j}=\beta_{j i},
$$

for $i, j \in\{K, L, E, M\}$ and $i \neq j$. Furthermore, because the cost shares must sum to one, we have to impose two additional restrictions, given by:

$$
\sum_{i} \beta_{i}=1
$$

and

$$
\sum_{j} \beta_{i j}=0 \text { and } \sum_{i} \beta_{i j}=0,
$$

for $i, j \in\{K, L, E, M\}$. These restrictions are derived in Christensen et al. (1973), and can be described as identifiability of the distribution parameters and Cournot aggregation, respectively (see Hanoch, 1975). The two restrictions are imposed in empirical research by dividing the first $N-1$ input prices by the price of the $N^{\text {th }}$ input, the choice for the latter being arbitrary. This system of share equations is a system of seemingly unrelated demand equations that is singular in its disturbances, which is solved by dropping the $N^{\text {th }}$ share equation. The resulting system of $N-1$ share equations is non-singular and can be estimated linearly. As an example we use a KLEM production function, where $s_{M}$ is the omitted share equation. The system to be estimated in this case looks as:

$$
s_{i}=\beta_{i}+\sum_{j} \beta_{i j} \ln \left(p_{j} / p_{M}\right)
$$

for $i, j \in\{K, L, E\}$. In most of the empirical literature either a SURE or an (iterative) 3SLS model is applied for estimation. The latter method is used to circumvent problems due to the possible endogeneity of input prices. Although the parameters that are obtained by estimating this system are usually not of much interest in themselves, they can be used to derive several substitution elasticity measures. This is the subject of the next section. 


\section{Cross-price and Morishima substitution elasticities}

Two generalisations of the original elasticity of substitution by Hicks (1932) are discussed in Allen and Hicks (1934). The first is Hicks' elasticity of substitution and consists of applying the two input formulae to each pair of inputs, holding other inputs constant. Obviously, this measure is biased because it does not allow for an optimal adjustment of all input factors to a change in a price ratio. The second concept was originally coined the partial elasticity of substitution, but is now generally known as the Allen elasticity of substitution (AES). It can be calculated from the system of translog cost share equations discussed in the previous section by (see Uzawa, 1962):

$$
A E S_{K E}=\left(\hat{\beta}_{K E}+s_{K} s_{E}\right) / s_{K} s_{E},
$$

where $\hat{\beta}_{K E}$ is an estimate obtained by estimating the system in equation (5). Specifically, it is an estimate of the Slutsky substitution elasticity. Furthermore, Allen (1938) has shown that the cross-price elasticity $(C P E)$ and the Allen elasticity of substitution are related according to $C P E_{K E}=s_{E} A E S_{K E}$, which shows that the AES and $C P E$ are always of the same sign, since $s_{E}$ is strictly positive. The cross-price elasticity can be now be derived from the estimated translog parameters by: ${ }^{4}$

$$
C P E_{K E}=\left(\hat{\beta}_{K E}+s_{K} s_{E}\right) / s_{K} \text {. }
$$

Although the $A E S$ and $C P E$ are relevant in their own right, they are one-input-one-price elasticities. Therefore, they do not measure changes in factor ratios as is the case in the original elasticity notion brought forward by Hicks (1932). Furthermore, although the $C P E$ and AES appear to be similar measures, the $C P E$ is to be preferred to the AES for several reasons. A first difference between the two measures is that the $A E S$ 's are symmetric, implying $A E S_{K E}=A E S_{E K}$, while the $C P E$ 's are not. Since substitution potential between capital and labour is most likely not identical to the substitution potential between energy and labour, resulting in a different adjustment mechanism for the respective price changes, symmetry of the cross-price elasticities of capital and energy is unrealistic. Second, the AES is more sensitive to factor shares than the CPE (see Berndt and Wood, 1981). In contrast to the AES, the $C P E$ is a scale-free elasticity measure, which is preferable when we want to compare the magnitude of the estimated elasticities between different studies. Finally, the $C P E$ exactly measures the percentage change in demand for factor $K$ due to a percentage change in the price of factor $E$. Therefore, the only possible function of the AES is to make the $C P E$ estimates symmetric; as discussed above, this is neither necessary nor preferable. Given these drawbacks and because each study that provides

\footnotetext{
${ }^{4}$ The own price elasticities are derived in a similar vein (see Uzawa, 1962).
} 
an $A E S$ estimate also provides a $C P E$ estimate (see also Blackorby and Russell, 1989), we pay no further attention to $A E S$ estimates in the remainder of this paper. ${ }^{5}$

In this paper we are also interested in measuring the purely technical potential for substitution between capital and energy. A measure first developed by Morishima (1967), later on independently discovered by Blackorby and Russell (1975), is what is now called the Morishima elasticity of substitution $(M E S)$. Assuming a change in $p_{E}$, while holding $p_{K}$ constant, the $M E S$ between $K$ and $E$ is given by (see Blackorby and Russell, 1989):

$$
M E S_{K E}=C P E_{K E}-P E_{E},
$$

where $P E_{E}$ is the own price elasticity of energy. A clear difference between the $C P E$ on the one hand and the $M E S$ on the other, is that the latter is a one-price-two-factor elasticity and, as such, measures exactly the curvature of the production isoquant, assuming a change in the price of one factor while keeping other prices constant. In mathematical terms it is easy to see that the $M E S$ is closely related to the original elasticity notion of Hicks. Note that we can write equation (8) as:

$$
M E S_{K E}=\frac{d \ln (K)}{d \ln \left(p_{E}\right)}-\frac{d \ln (E)}{d \ln \left(p_{E}\right)}=\frac{d \ln (K / E)}{d \ln \left(p_{E}\right)}
$$

which is the required two-factor-two-price measure because $p_{K}$ is constant, implying that a percentage change in the $p_{E} / p_{K}$ price ratio reduces to a percentage change in $p_{E}$ only. The differences between the two elasticity measures notwithstanding, both the $C P E$ and the $M E S$ allow for a natural asymmetry in the elasticity of substitution of an input combination. ${ }^{6}$

Since $P E_{E}$ should be negative, note from equation (8) that the $C P E$ and the $M E S$ differ in sign when $C P E_{K E}$ is negative and $\left|P E_{E}\right|>\left|C P E_{K E}\right|$. These conditions are by no means unlikely and the difference between the two elasticity measures is therefore not trivial. In addition, whereas the $C P E$ can be negative since demand for $K$ may actually decline when the price of $E$ increases, the $M E S$ cannot be negative. To see this, remember that the $M E S$ unit of measurement is a percentage change in the $(K / E)$ ratio instead of a percentage change in demand for $K$. A negative $M E S$ would therefore mean that an increase in the price of energy causes a decline in demand for capital that is larger than the decline in

\footnotetext{
${ }^{5}$ Either the $C P E$ estimate is given in the study itself, or we were able to derive it from the $A E S$ estimate. In the latter case the necessary information on factor shares was always given or could be derived from the study.

${ }^{6}$ An alternative measure is the shadow elasticity of substitution, which is simply a weighted average - with factor shares as weights - of the two Morishima substitution elasticities (see Nguyen and Streitwieser, 1999). This measure is excluded from our analysis since it imposes symmetry of the substitution elasticities.
} 
demand for energy. This would imply a substitution away from capital despite the fact that the relative price of capital has decreased.

As already mentioned, the $M E S$ is close to the original notion of a substitution elasticity in that it measures the percentage change in a factor ratio. In contrast, a $C P E$ measures solely the actual percentage change in demand for $K$ due to a change in $p_{E}$. Note that this actual change consists of two separate effects, i.e., a substitution and an income effect. The substitution effect measures a direct change in demand for $K$ due to a change in $p_{E}$, while the income effect measures a change in demand for $K$ due to a change in income brought about by a change in $p_{E}$. The difference between the cross price elasticity and the Morishima elasticity of substitution is exactly this income effect. ${ }^{7}$ Since the income effect is most likely negative, the cross price elasticity is lower than the substitution elasticity. Moreover, the two measures may also differ in sign. This is the case when the substitution effect is positive but the income effect dominates the substitution effect in absolute terms. Which measure to prefer depends primarily on the research question. Investigations on energy policy plans require information on actual changes, while academic studies on for instance engineering substitution potential in different sectors of the economy are likely to be more interested in Morishima measures. In conclusion, in this paper we focus on cross price elasticities and Morishima substitution elasticities, and exclude Allen elasticities, Shadow elasticities, and nested CES estimates from our analysis.

\section{Sample and study characteristics}

This section describes our sampling procedure and the database underlying our meta-analysis. Our method of searching for and sampling empirical studies on capital-energy substitution is fairly straightforward. We first looked for relevant studies by making use of standard online search engines, such as Econlit and Picarta. We subsequently searched for relevant studies in the reference lists of the articles that were collected in the first step. We excluded studies for developing countries from the database for two reasons. First, production processes and available energy saving technologies in these countries can be expected to be very different from those in more modernised parts of the world. A second and more pragmatic reason is that many of the existing studies for these countries could not be attained.

It is important to note that many studies do not report $M E S$ estimates. In order to increase the number of observations in our dataset we calculated $M E S$ estimates for many studies ourselves, using the own and cross price elasticities and the relation in equation (8). Standard errors for these estimates were calculated using the Delta method (see Greene, 2000, pp. 357-360). In Appendix A we pay de-

\footnotetext{
${ }^{7}$ The distinction between cross price and Morishima substitution elasticities is sometimes also referred to as the difference between engineering and economic measures of substitution (see Berndt and Wood, 1979; Prywes, 1986).
} 
tailed attention to the specific procedure. If standard errors are not available and could not be calculated because the information provided in a study was insufficient, the elasticity is excluded from the dataset. ${ }^{8}$ Given these sampling restrictions, we ultimately arrive at two different samples with the following characteristics:

(I) A sample with MES elasticities on substitution of capital for energy, consisting of 156 observations from 21 different studies $\left(M E S_{K E}\right)$;

(II) A sample with $C P E$ elasticities on the change in the demand for capital, consisting of 155 observations from 20 different studies $\left(C P E_{K E}\right)$.

In Appendix B we give a detailed description of each study included in the meta-analysis. Except for the study by Falk and Koebel (1999), which applies a quadratic cost function, all primary studies in the sample apply a translog production function. Studies that use other flexible functional forms, such as the Generalised Leontief and the Generalised Cobb Douglas specifications, provide too little information to calculate standard errors of the elasticity estimates. There is also wide variation in other production function characteristics, such as the inclusion of labour and materials in the model specification and the inclusion of returns to scale and non-neutral technological change parameters. Although most studies use data for the USA, other data characteristics, such as the level of data aggregation and the use of cross section, time series or panel data, differ widely between studies.

In order to get a good overall picture of the distribution of estimates we present $M E S$ and $C P E$ elasticities and their 95\% confidence intervals in Figure 1 and Figure 2, respectively. The substitution or Morishima elasticities display more variation than the cross price elasticities, ranging from 0 to approximately 4 . In the MES sample a substantial proportion of estimates is larger than one, implying elastic technical substitution. Because we excluded negative estimates that are statistically different from zero at 5\%, the few negative elasticity estimates that are present in the MES sample are statistically indistinguishable from zero. The figures reveal to a certain extent why the discussion on capitalenergy substitution has become so confusing over the years. Around 35\% of the CPE estimates is negative and all estimates are between -1 and 1. Interpreting $C P E$ estimates as measures of technical substitution potential therefore gives a diffuse answer to the question whether capital and energy are substitutes or complements.

\footnotetext{
${ }^{8}$ Furthermore, although a negative cross price elasticities is theoretically valid, a negative Morishima elasticity is not (see Section 3). Therefore we exclude $M E S$ elasticities that are negative and statistically different from zero. The $C P E$ elasticities that are used to calculate these particular MES estimates are also excluded, simply because these estimates are not reliable. We furthermore excluded elasticities for which it is clear that they hold for working capital only, since we are interested in substitution between actual machinery and energy.
} 


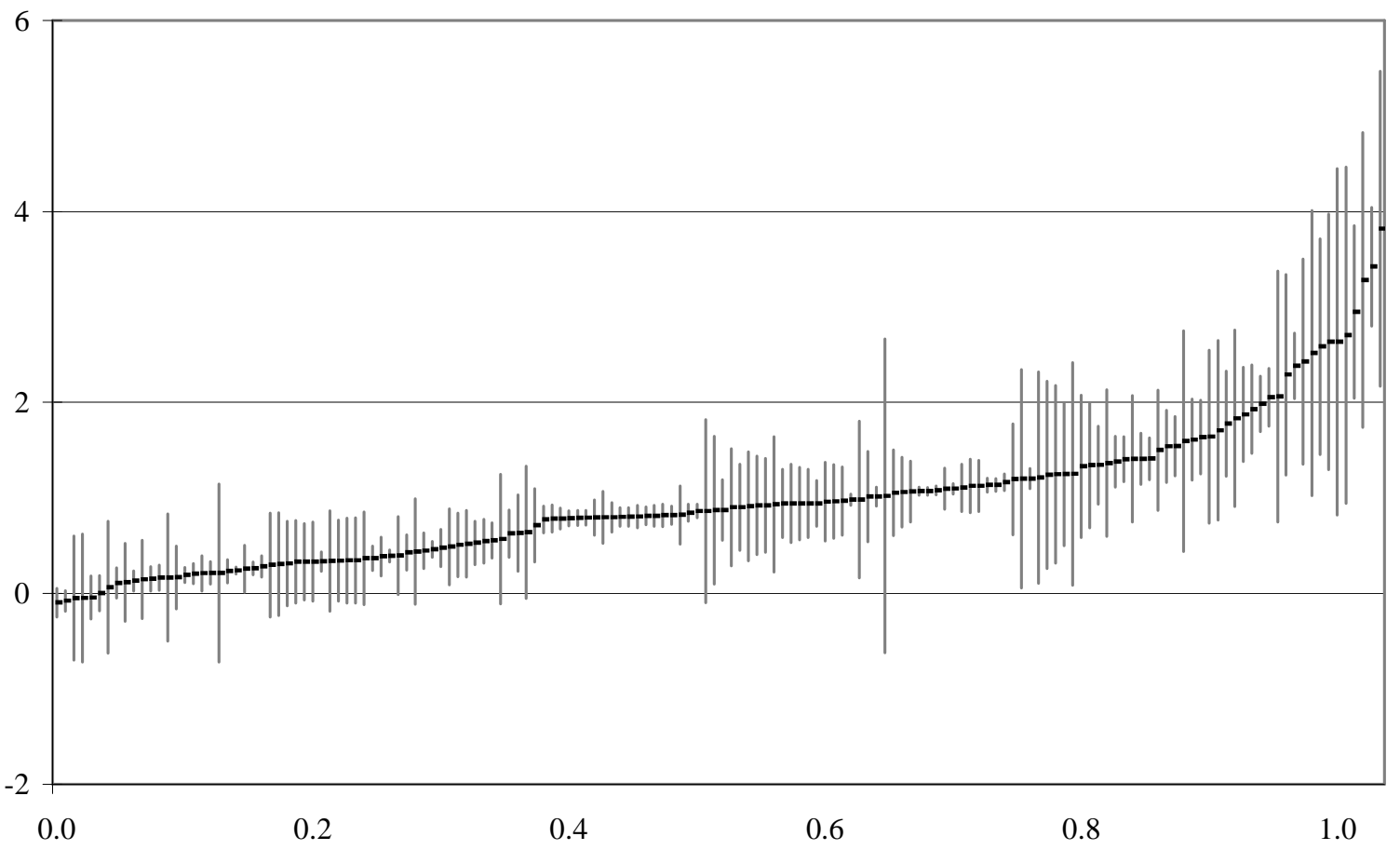

Figure 1: $M E S_{K E}$ elasticities and their $95 \%$ confidence intervals (elasticity values on the vertical axis and sample size deciles on the horizontal axis)

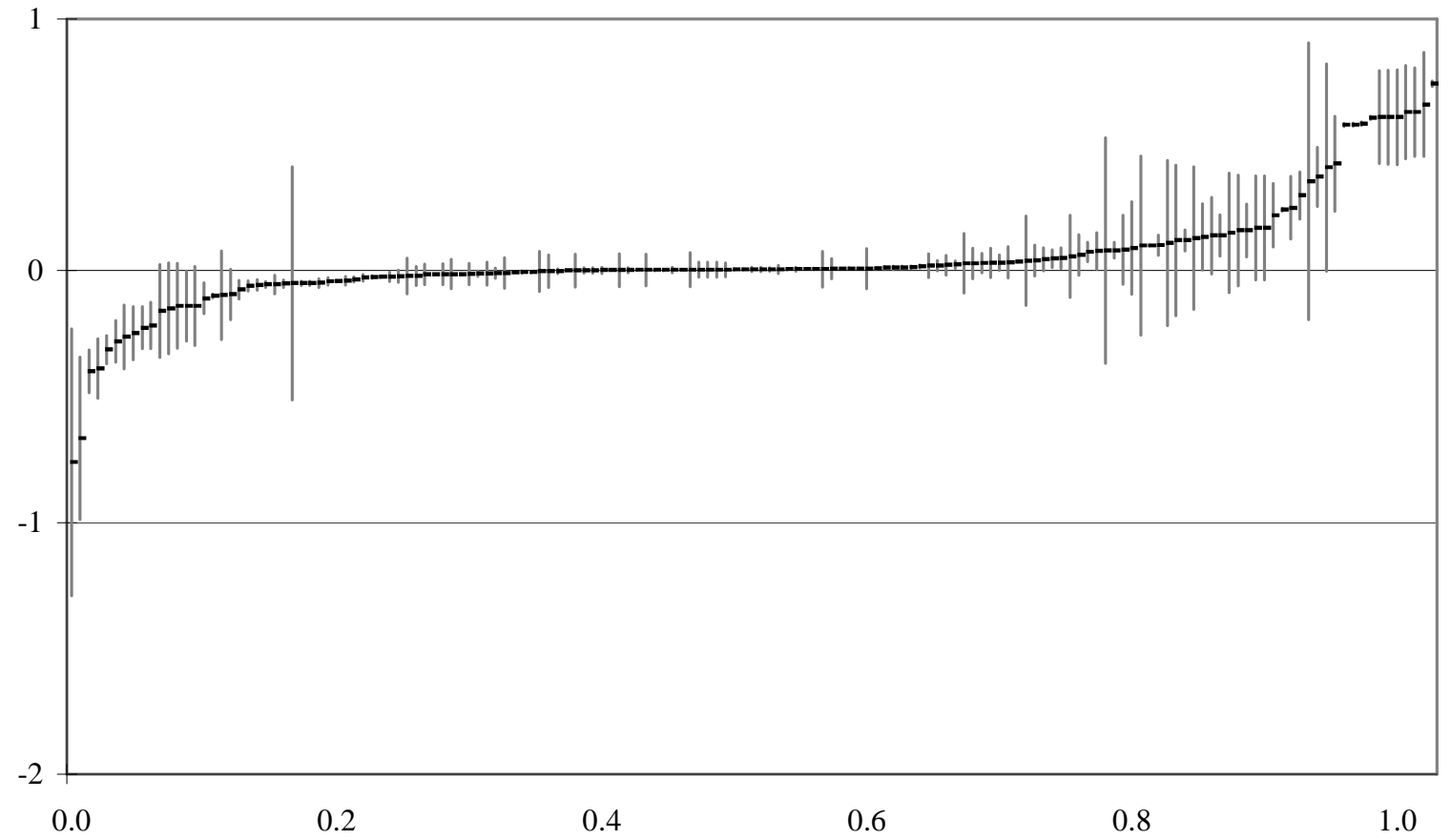

Figure 2: $C P E_{K E}$ elasticities and their $95 \%$ confidence intervals (elasticity values on the vertical axis and sample size deciles on the horizontal axis) 
For an overall statistical estimate for the two elasticities we may revert to fixed and random effects models (see Sutton et al., 2000). A potential disadvantage of these models is that they are univariate and do not account for possible systematic variation in elasticity estimates, for instance due to differences in primary study characteristics. In order to test for this we use the $Q$-statistic, which measures whether primary study estimates in a meta-analysis represent a single population effect size. It is given by (see Sutton et al., 2000, p. 38):

$$
Q=\sum_{i=1}^{I} \frac{1}{\sigma_{i}^{2}}\left(\eta_{i}-\eta\right)^{2}
$$

where $\eta_{i}$ are the estimated elasticities (either substitution or cross price elasticities), $\sigma_{i}^{2}$ represent the variances of the elasticities, $\eta$ is the fixed effect as estimated by the fixed effects meta-model, which is given by (Hedges, 1994):

$$
\eta=\sum_{i=1}^{I} \eta_{i}\left(\sigma_{i}^{2}\right)^{-1} / \sum_{i=1}^{I}\left(\sigma_{i}^{2}\right)^{-1},
$$

and $i=1,2, \ldots, I$ is an elasticity index. The $Q$-statistic is $\chi^{2}$ distributed with $I-1$ degrees of freedom. In both samples most primary studies provide more than one effect size for our meta-analysis; this poses a problem that is generally referred to as multiple sampling. One way to account for this is to weight each effect size by the total number of effect sizes drawn from the same study. This approach ensures that each study instead of each observation gets an equal weight. An alternative to solve the multiple sampling problem is to use a single sampling set-up, e.g., by including only the median of the effect sizes from a single study.9 This approach prevents sampling issues driving the results - as is the case when we sample a single effect size randomly from the sample - and has the advantage that the standard error is readily available - in contrast to the situation in which we use the average of the effect sizes from a single study. We calculate the Q-statistic using the three approaches mentioned above, i.e., the multiple sampling model with both equal weight per observation and equal weight per study, and the single sampling model. The calculated $Q$-statistics are statistically significant at $5 \%$ in all cases, implying that the effect sizes in each sample do not represent a single underlying population effect size. The fixed and random effects estimates are therefore unreliable and probably biased estimates of substitution potential between capital and energy. In the next section we therefore discuss possible sources of systematic effect size variation. In our meta-regression model we subsequently account for these factors in order to uncover which of them are indeed empirically relevant.

\footnotetext{
${ }^{9}$ When the amount of effect sizes obtained from a study is an even number, we take the effect size with the lowest absolute value to make sure we do not overestimate the meta effects.
} 


\section{Sources of systematic effect size variation}

In this section we discuss differences between primary studies on capital-energy substitution may cause systematic variation estimated substitution elasticities. The differences between primary studies are manifold. For instance, a discussion in the 1970s on the differences in empirical substitution estimates between Berndt and Wood (1979) and Griffin and Gregory $(1976,1981)$ revolves around two central issues. Griffin and Gregory claim that the prevailing differences are related to differences between short run measures from time series data and long run measures from cross section data. The ar-

gument here is that time series data do not incorporate long run factor adjustments, and that elasticity measures from time series studies should therefore be considered short run elasticities. In contrast, it is likely that cross section data do incorporate these long run adjustments. In a reaction to this claim, Berndt and Wood (1979) state that it is more likely that differences between gross and net substitution measures cause the observed variation in empirical substitution estimates. Their argument revolves around the issue of separability of production factors. In many production studies using flexible functional forms, materials are excluded as an input factor. Implicitly or explicitly, this is equivalent to the assumption that capital, labour and energy are separable from materials in a production process. However, when separability does not hold in reality, the estimated production function parameters are most likely biased. Our meta-analysis includes a distinction between different types of data used in primary studies, and between studies that include and do not include materials as an input factor in production.

Other important issues in estimating a production function are assumptions with respect to returns to scale and neutral or non-neutral technological change. In estimating a translog cost function many empirical studies assume a homothetic, constant returns to scale production technology and absence of non-neutral technological change. This may be problematic since parameter estimates may be biased when these assumptions do not hold in reality. Some studies therefore explicitly use a translog cost function incorporating returns to scale and technological change parameters. The system of equations in this case looks as:

$$
s_{i}=\beta_{i}+\sum_{j} \beta_{i j} \ln \left(p_{j} / p_{M}\right)+\beta_{i y} \ln y+\beta_{i t} t
$$

for $i, j \in\{K, L, E\}$, where $y$ is output, $t$ is a time index, and $\beta_{i y}$ and $\beta_{i t}$ are parameters to be estimated. In addition we need the parameter restrictions:

$$
\sum_{i} \beta_{i y}=\sum_{i} \beta_{i t}=0
$$


for the cost function to be homogeneous in input prices. Homotheticity implies that relative factor shares are independent of the level of output. A necessary and sufficient condition for a production technology to be homothetic is that $\beta_{i y}=0$ for all $i$.

Differences in production function estimates may be caused by differences in data aggregation, which may take several forms. First, many studies use industry wide data, which potentially disguises the fact that capital-energy substitution potential differs between sectors. Another problem with using aggregate data is that substitution patterns obtained from these studies may be caused by changes in the composition of production at the sectoral level. For instance, the composition of production in a certain sector may shift over time towards the production of more capital- or more energy-intensive products. Although driving forces of substitution may be identical, factor substitution due to shifts in sectoral production composition is clearly not the same as substitution along the production isoquant of an individual firm. Moreover, in extreme cases, substitution at higher levels of data aggregation may disguise actual factor complementarity at the firm level. A fortiori, since changes in a production process at the firm level are limited when input factors are complements, changes in relative factor prices may in fact induce changes in the composition of production at the sectoral level. Especially when relative price changes are permanent, the production of products that use less of the more expensive input factor has become more attractive. Therefore, complementarity at the firm level may induce substitution at the sectoral level. Although we can distinguish between studies that use disaggregate data and studies that use aggregate data, the potential trade-off between firm level factor complementarity on the one hand, and factor substitution at the sectoral level on the other, cannot be identified in our empirical analysis.

Second, other aggregation issues revolve around the potentially important distinction between different kinds of labour and different types of energy sources. Similar to aggregation of different types of capital, aggregation of energy sources may disguise substitution patterns that take place at lower levels of aggregation. Most studies use aggregate energy data, but some studies distinguish between oil, gas and electricity. Compared to aggregate energy elasticity measures, our analysis allows us to analyse whether the relation between capital and energy is different for different types of energy. Furthermore, labour may be subdivided into skilled and unskilled labour (see, among others, Halvorsen and Ford, 1979; Falk and Koebel, 1999), a possible hypothesis being that the substitution potential between capital and skilled labour is smaller than the substitution potential between capital and unskilled labour. Obviously, when such a distinction does not affect the estimated production function parameter estimates we do not have a problem. However, a priori there is no reason to suspect that erroneous aggregation of labour does not lead to biased or otherwise flawed production function estimates. 
Third, although primary studies differ slightly with respect to the measurement of labour and energy, differences in the measurement of capital are of special interest. As argued in Field and Grebenstein (1980), differentiation between physical capital and working capital is a potentially important source of variation in empirical estimates of capital-energy substitution. Physical capital refers to actual machinery, while working capital refers to other types of capital, such as buildings and structures, land, and monetary assets. Some studies distinguish explicitly between physical capital and working capital. As discussed in Section 4, we exclude elasticities for which it is clear that they hold for working capital only, since we are interested in substitution between actual machinery and energy. We explicitly distinguish empirical estimates that are estimated for physical capital only, as opposed to estimates that hold for an aggregate capital measure. ${ }^{10}$

Finally, there are a number of remaining issues. For instance, as the energy saving capacity of technologies has evolved over time, we may observe a temporal shift in capital-energy substitutability. As an example, Ilmakunnas and Törmä (1989) estimate a model with a change in the structural parameters. They find a shift from capital-energy complementarity in the period 1960-1973 to capitalenergy substitutability in the period 1974-1981. Although most primary studies do not explicitly test for structural shifts in the elasticity measure, including a time trend parameter in our empirical analysis may reveal a temporal pattern in estimated substitution potential. Furthermore, factor prices may be endogenous. We therefore create a dummy variable for studies that control for possible endogeneity by using instrumental variables in their model estimations. Finally, although most primary studies use aggregate manufacturing data, a fairly substantial number of estimates is available for the chemical and the metal industry.

With the exception of the time trend parameter, we create dummy variables for each specific primary study characteristic. These dummy variables are included as explanatory variables in our meta-model specification in order to analyse whether they represent relevant sources of effect size variation. They can be subdivided into four main categories, i.e., omitted variables, data characteristics, aggregation of explanatory variables, and remaining issues. A description of the variables included in the meta-model is given in Table 1.

\footnotetext{
${ }^{10}$ Another potential source of variation in study outcomes is related to the fact that some studies allow for substitution between different types of energy (see Fuss, 1977; Pindyck, 1979; Turnovsky and Donnelly, 1984). However, the energy sub-model is always estimated separately from the aggregate production function, implying that substitution between different types of energy should not (substantially) affect the estimated substitution elasticities between capital and energy. Since the 'energy subsystem dummy variable' is highly correlated with the returns to scale dummy, we exclude the former from our analysis.
} 
Table 1: Description and operationalisation of explanatory variables in the meta-model

\begin{tabular}{|c|c|}
\hline Variable & Description and operationalisation \\
\hline \multicolumn{2}{|l|}{ Omitted variables } \\
\hline Materials excluded & $\begin{array}{l}\text { Dummy variable equal to one when materials are not included among the ex- } \\
\text { planatory variables in a primary model. }\end{array}$ \\
\hline Returns to scale & $\begin{array}{l}\text { Dummy variable equal to one when the primary model allows for non-constant } \\
\text { returns to scale, i.e., includes returns to scale parameters. }\end{array}$ \\
\hline $\begin{array}{l}\text { Non-neutral techno- } \\
\text { logical change }\end{array}$ & $\begin{array}{l}\text { Dummy variable equal to one when the primary model includes non-neutral } \\
\text { technological change parameters. }\end{array}$ \\
\hline \multicolumn{2}{|l|}{ Data characteristics } \\
\hline 2- or 4-digit data & $\begin{array}{l}\text { Dummy variable equal to one when a study uses } 2 \text { - or 4-digit manufacturing } \\
\text { data, opposed to 1-digit manufacturing data. }\end{array}$ \\
\hline Cross section data & Dummy variable equal to one when a primary study uses cross section data. \\
\hline Panel data & Dummy variable equal to one when a primary study uses panel data. \\
\hline \multicolumn{2}{|c|}{ Aggregation of explanatory variables } \\
\hline Fuel energy & $\begin{array}{l}\text { Dummy variable equal to one when substitution between capital and fuel en- } \\
\text { ergy (opposed to aggregate energy) is estimated. }\end{array}$ \\
\hline Electric energy & $\begin{array}{l}\text { Dummy variable equal to one when substitution between capital and electric } \\
\text { energy (opposed to aggregate energy) is estimated. }\end{array}$ \\
\hline $\begin{array}{l}\text { Labour disaggre- } \\
\text { gated }\end{array}$ & $\begin{array}{l}\text { Dummy variable equal to one when a primary study makes a distinction be- } \\
\text { tween different types of labour, usually between skilled and unskilled labour. }\end{array}$ \\
\hline Machinery & $\begin{array}{l}\text { Dummy variable equal to one when an elasticity between energy and machin- } \\
\text { ery (opposed to aggregate capital) is estimated. }\end{array}$ \\
\hline \multicolumn{2}{|l|}{ Remaining issues } \\
\hline $\begin{array}{l}\text { Instrumental vari- } \\
\text { ables }\end{array}$ & $\begin{array}{l}\text { Dummy variable equal to one when the primary model is not estimated with in- } \\
\text { strumental variables (3SLS). }\end{array}$ \\
\hline Chemical industry & $\begin{array}{l}\text { Dummy variable equal to one when the elasticity was specifically estimated for } \\
\text { the chemical industry. }\end{array}$ \\
\hline Metal industry & $\begin{array}{l}\text { Dummy variable equal to one when the elasticity was specifically estimated for } \\
\text { the metal industry. }\end{array}$ \\
\hline Year of evaluation & $\begin{array}{l}\text { The year evaluation is either explicitly given in the study or taken as the middle } \\
\text { year of the data period used in the primary model estimation. }\end{array}$ \\
\hline
\end{tabular}

\section{6. $\quad$ Estimation procedure and results}

In this section we present the results of a meta-regression analysis for the Morishima and cross price elasticity samples. An important methodical issue in meta-analysis is effect size heterogeneity due to heterogeneity in effect size variance and the possibility that the true underlying effect varies randomly across primary studies. Ignoring these issues may increase the variance of the meta-estimator and may bias the standard errors of the estimated coefficients (see Koetse, 2006, Chapter 5). The standard approach to deal with heterogeneity in effect size variance is to weight the dependent and independent variables in the meta-analysis by the standard errors of the elasticities obtained from the primary studies. The fact that the true underlying effect size may vary randomly across primary studies is generally addressed by explicitly estimating the variance of the population of random effect sizes and including 
this variance in the weights. The resulting model, with fixed effects on potential sources of systematic effect size variation and weights defined by the sum of the effect size variance and the variance of the population of random effect sizes, is referred to as the mixed effects model (Hedges and Olkin, 1985). Finally, we deal with multiple sampling by giving each study, instead of each observation, an equal weight in our estimations. ${ }^{11}$ We apply maximum likelihood to estimate the resulting model.

The results of the meta-regression analysis are presented in Table 2. We estimate separate parameters for the $M E S$ and the $C P E$ samples and also present the differences between these estimates. Given the operationalisation of the explanatory variables, the constant in each model represents the estimated elasticity for a $K L E$ production function that does not include returns to scale and non-neutral technological change parameters, that is estimated by a model that does not account for possible endogeneity in (one of) the explanatory variables, that uses aggregate capital, labour and energy data, and 1-digit manufacturing time series data. The results are presented in Table 2.

When comparing the results the differences between the MES and CPE coefficients stand out, especially for the constant and for the coefficients on 'control variables in primary models'. The results suggest that, in contrast to a claim made by Berndt and Wood (1979), capital and energy are separable from materials in production functions, but only in a technical sense. Since the income effect is negative, separability does not hold for cross price elasticities. Specifically, demand for energy and capital decreases when total income decreases due to materials price changes. Therefore, if materials prices are excluded from primary models, changes in energy and capital demand may be erroneously related to variation in the prices of input factors other than materials, thereby producing biased estimates of capital-energy cross price elasticities.

Table 2: Mixed effects estimations for Morishima substitution and cross price elasticities on substitution of capital for energy; equal weights per study (standard errors in parentheses)

\begin{tabular}{l|c|c:c}
\hline Meta-model & \multicolumn{2}{|c}{ Mixed effects model with equal weights per study } \\
\hline Dependent variable & Morishima elasticities & Cross price elasticities & Difference \\
\hline \hline Constant & $.264^{*}$ & $-.061^{*}$ & $-.324^{*}$ \\
& $(.110)$ & $(.025)$ & $(.116)$ \\
& Control variables in primary models & \\
Materials excluded & .039 & $-.092^{*}$ & $-.132^{*}$ \\
& $(.055)$ & $(.034)$ & $(.062)$ \\
Returns to scale & -.111 & $.226^{*}$ & $.337^{*}$ \\
& $(.128)$ & $(.031)$ & $(.132)$ \\
Non-neutral technologi- & $.380^{*}$ & $.193^{*}$ & $-.187^{*}$ \\
cal change & $(.076)$ & $(.043)$ & $(.083)$ \\
\hline
\end{tabular}

\footnotetext{
${ }^{11}$ Another way to deal with this issue would be to estimate a hierarchical level model (see, e.g., Goldstein and Rasbash, 1992).
} 
Table 2: Continued

\begin{tabular}{|c|c|c|c|}
\hline Meta-model & \multicolumn{3}{|c|}{ Mixed effects model with equal weights per study } \\
\hline Dependent variable & Morishima elasticities & Cross price elasticities & Difference \\
\hline \multicolumn{4}{|c|}{ Data characteristics } \\
\hline 2-digit or 4-digit data & $\begin{array}{l}.221 \\
(.149)\end{array}$ & $\begin{array}{l}-.035 \\
(.031)\end{array}$ & $\begin{array}{l}-.255 \\
(.161)\end{array}$ \\
\hline Panel data & $\begin{array}{l}.248^{*} \\
(.103)\end{array}$ & $\begin{array}{l}-.116^{*} \\
(.049)\end{array}$ & $\begin{array}{l}-.363^{*} \\
(.115)\end{array}$ \\
\hline Cross section data & $\begin{array}{l}.570^{*} \\
(.210)\end{array}$ & $\begin{array}{l}.351^{*} \\
(.073)\end{array}$ & $\begin{array}{l}-.219 \\
(.228)\end{array}$ \\
\hline \multicolumn{4}{|c|}{ Aggregation of explanatory variables } \\
\hline Fuel energy & $\begin{array}{l}.756^{*} \\
(.294)\end{array}$ & $\begin{array}{c}.507^{*} \\
(.034)\end{array}$ & $\begin{array}{l}-.248 \\
(.311)\end{array}$ \\
\hline Electric energy & $\begin{array}{l}.243 \\
(.188)\end{array}$ & $\begin{array}{l}.033 \\
(.034)\end{array}$ & $\begin{array}{l}-.209 \\
(.195)\end{array}$ \\
\hline Labour disaggregated & $\begin{array}{c}.138 \\
(1.427)\end{array}$ & $\begin{array}{l}-.276^{*} \\
(.071)\end{array}$ & $\begin{array}{l}-.414 \\
(1.443)\end{array}$ \\
\hline Machinery & $\begin{array}{l}.063 \\
(.083)\end{array}$ & $\begin{array}{l}.085^{*} \\
(.042)\end{array}$ & $\begin{array}{c}.022 \\
(.091)\end{array}$ \\
\hline \multicolumn{4}{|c|}{ Remaining issues } \\
\hline Instrumental variables & $\begin{array}{l}.110 \\
(.081)\end{array}$ & $\begin{array}{l}.000 \\
(.030)\end{array}$ & $\begin{array}{l}-.110 \\
(.093)\end{array}$ \\
\hline Chemical industry & $\begin{array}{l}.177 \\
(.528)\end{array}$ & $\begin{array}{l}-.043 \\
(.058)\end{array}$ & $\begin{array}{l}-.219 \\
(.492)\end{array}$ \\
\hline Metal industry & $\begin{array}{l}.028 \\
(.481)\end{array}$ & $\begin{array}{l}-.002 \\
(.052)\end{array}$ & $\begin{array}{l}-.031 \\
(.464)\end{array}$ \\
\hline Year of evaluation & $\begin{array}{l}-.001 \\
(.004)\end{array}$ & $\begin{array}{l}.002^{*} \\
(.001)\end{array}$ & $\begin{array}{l}.003 \\
(.004)\end{array}$ \\
\hline $\begin{array}{l}\text { Root of between study } \\
\text { variance }\end{array}$ & $\begin{array}{l}.000 \\
(.008)\end{array}$ & $\begin{array}{l}.007^{*} \\
(.001)\end{array}$ & $\begin{array}{l}.007 \\
(.008)\end{array}$ \\
\hline $\begin{array}{l} \\
\text { Mean Log-L } \\
\text { Restricted Mean Log-L }\end{array}$ & $\begin{array}{r}156 \\
-.843 \\
-1.229\end{array}$ & $\begin{array}{r}155 \\
.749 \\
-.200\end{array}$ & $\begin{array}{r}311 \\
-.050 \\
-.941\end{array}$ \\
\hline
\end{tabular}

$*=$ Statistically significant at $5 \%$

The results for the MES sample confirm the claim made by Griffin and Gregory (1981) that the use of cross section opposed to time series data leads to higher elasticity estimates. Although our results do not unveil the reasons for this result, their reasoning is that cross section data, in contrast to time series data, reflect long run changes in factor demand. The use of panel data leads to intermediate estimates of substitution, which makes sense since both short and long run changes and processes are incorporated in this type of data. The findings for cross price elasticities show that the income effect domi- 
nates the substitution effect in the short run, but that capital-energy substitution potential increases as time progresses, resulting in an increase in actual demand for capital in the long run. On the other hand, studies using panel data produce lower cross price elasticities than studies using time series data. Given the increase in technical substitution potential indicated by the results for the MES sample, this suggests that the income effect also increases over time. Therefore, the behaviour of short, medium and long run cross price elasticities is determined by the development of the relative magnitude of the substitution and income effect. Apparently, the income effect dominates the substitution effect in the short run and a fortiori in the medium run. However, in the long run this pattern is reversed, thereby increasing the actual demand for capital as time progresses.

Returns to scale and technological change parameters are important control variables in production functions. Although the inclusion of returns to scale parameters in a production function does not have a systematic effect on the estimated Morishima elasticities, it has a substantial systematic positive and statistically significant impact on the estimated cross price elasticity. Not including nonneutral technological change parameters in the production may substantially affect both the estimated Morishima and cross price elasticity. Increased demand for capital due to capital enhancing technological change is erroneously related to changes in energy prices when technological change parameters are not included in the production function.

Finally, results for both samples indicate that substitution between fuels and capital is higher than substitution between capital and other energy sources. The reason for this finding is most likely that fuel prices are highly volatile, mainly due to supply shocks. The uncertainty on future energy costs associated with this volatility may induce a firm to concentrate on investing in energy saving technologies. Judging by their statistical significance, labour and capital disaggregation, the use of instrumental variables, the estimation of sector specific elasticities, and the year of evaluation in the primary study do not contribute to explaining the variation in the MES sample. The latter result is somewhat surprising. Because of the increasing availability and increasing performance of energy sav-

ing technologies over time we would expect a positive time trend in substitution potential. However, the bulk of the estimates is estimated in the seventies and eighties, and the small number of observations throughout the nineties is too small to warrant a strong conclusion on the development of capitalenergy substitution potential during more recent periods.

\section{Discussion}

In this paper we presented the results of a meta-regression analysis for estimated elasticities on capitalenergy substitution. Although in most cases the results of a meta-analysis do not give decisive insights into the correctness of certain theories, assumptions and model specifications, our findings clearly reveal the relevant sources of omitted variable and aggregation bias in primary studies. We can therefore 
sensibly compute ideal-typical Morishima and cross price elasticities for the short, the medium and the long run. An ideal-typical elasticity is defined as the elasticity that results when it would be estimated under ideal circumstances (which, of course, is subjective to a certain extent). In order to arrive at such ideal-typical elasticities we have to take the sum of the constant and the relevant coefficients. ${ }^{12} \mathrm{We}$ only include coefficients from the meta-regression analysis that are statistically significant. Specifically, short run Morishima elasticities are elasticities from KLEM production functions that include non-neutral technological change parameters and use time series data. The short run version of the ideal-typical cross price elasticity is an elasticity from a KLEM production function that includes both returns to scale and non-neutral technological change parameters, and uses time series data and disaggregated capital and labour data. The medium and long run versions differ from their short run counterparts in that they are calculated for primary studies that use panel and cross section data, respectively. The resulting ideal-typical elasticities, along with their $95 \%$ confidence intervals, are presented in Table 3.

Table 3: Ideal-typical Morishima and cross price elasticities and accompanying 95\% confidence intervals for the short, medium and long run

\begin{tabular}{|c|c|c|c|c|c|c|}
\hline \multirow{3}{*}{$\begin{array}{l}\text { Substitution elasticity } \\
\text { Confidence interval (95\%) }\end{array}$} & \multicolumn{2}{|c|}{$\begin{array}{c}\text { Short run } \\
\text { (time series data) }\end{array}$} & \multicolumn{2}{|c|}{$\begin{array}{l}\text { Medium run } \\
\text { (panel data) }\end{array}$} & \multicolumn{2}{|c|}{$\begin{array}{c}\text { Long run } \\
\text { (cross section data) }\end{array}$} \\
\hline & \multicolumn{2}{|c|}{.64} & \multicolumn{2}{|c|}{.89} & \multicolumn{2}{|c|}{1.21} \\
\hline & .40 & .89 & .64 & 1.14 & .70 & 1.73 \\
\hline Cross price elasticity & \multicolumn{2}{|c|}{.17} & \multicolumn{2}{|c|}{.05} & \multicolumn{2}{|c|}{.52} \\
\hline Confidence interval (95\%) & -.04 & .37 & -.09 & .20 & .27 & .77 \\
\hline
\end{tabular}

The numbers in the table clearly show that substitution elasticities are substantially higher than cross price elasticities, both in the short and in the long run. Therefore, despite the fact that technical opportunities to substitute capital for energy are considerable, they are almost entirely outweighed by the negative income effect brought about by energy price increases in the short and medium run; the short and medium run cross price elasticities are not statistically different from zero. In the long run this pattern does not hold. Our findings therefore suggest that increases in energy prices stimulate factor substitution but that this is a long run process. Actual changes in the demand for (energy saving) capital due to energy price increases take time.

\footnotetext{
${ }^{12}$ The standard error of such a composite elasticity is obtained by applying the Delta method (see Greene, 2000, pp. 357-358).
} 


\section{Acknowledgements}

This paper is written in the context of the program 'Stimulating the Adoption of Energy-Efficient Technologies', funded by the Netherlands Organisation for Scientific Research (NWO) and the Dutch Ministry of Economic Affairs (SenterNovem).

\section{References}

Allen RGD. Mathematical Analysis for Economists. MacMillan \& Co.: London; 1938.

Allen RGD, Hicks JR. A Reconsideration of the Theory of Value, Pt. II. Economica 1934; 1; 196-219.

Anderson RG 1981. On the Specification of Conditional Factor Demand Functions in Recent Studies of U.S. Manufacturing. In: Berndt ER, Field BC (Eds), Modeling and Measuring Natural Resource Substitution. MIT Press: Cambridge, Massachusetts; 1981. p. 119-144.

Andrikopoulos AA, Brox JA, Paraskevopoulos CC. Interfuel and Interfactor Substitution in Ontario Manufacturing, 1962-82. Applied Economics 1989; 21; 1667-1681.

Ball VE, Chambers RG. An Economic Analysis of Technology in the Meat Products Industry. American Journal of Agricultural Economics 1982; 64; 699-709.

Berndt ER. Aggregate Energy, Efficiency, and Productivity Measurement. Annual Review of Energy $1978 ; 3 ; 225-273$

Berndt ER, Wood DO. Technology, Prices, and the Derived Demand for Energy. Review of Economics and Statistics $1975 ; 57 ; 259-268$.

Berndt ER, Wood DO. Engineering and Econometric Interpretations of Energy-Capital Complementarity. American Economic Review 1979; 69; 342-354.

Berndt ER, Wood DO. Engineering and Econometric Interpretations of Energy-Capital Complementarity: Reply and Further Results. American Economic Review 1981; 71; 1105-1110.

Blackorby C, Russell RR. Will the Real Elasticity of Substitution Please Stand Up? (A Comparison of the Allen/Uzawa and Morishima Elasticities). American Economic Review 1989; 79; 882-888.

Blackorby C, RR Russell, 1975, The Partial Elasticity of Substitution, San Diego, San Diego.

Chang KP. Capital-Energy Substitution and the Multi-Level CES Production Function. Energy Economics 1994; 16; 22-26.

Christensen LR, Jorgenson DW, Lau LJ. Transcendental Logarithmic Production Function Frontiers. Review of Economics and Statistics 1973; 55; 28-45.

Dargay JM. The Demand for Energy in Swedish Manufacturing Industries. Scandinavian Journal of Economics 1983; 85; 37-51.

Debertin DL, Pagoulatos A, Aoun A. Impacts of Technological Change on Factor Substitution Between Energy and other Inputs within US Agriculture, 1950-79. Energy Economics 1990; 12; 210 .

Diewert WE, 1973, Separability and a Generalisation of the Cobb-Douglas Cost, Production and Indirect Utility Functions

Diewert WE. An Application of the Shephard Duality Theorem: A Generalised Leontief Production Function. Journal of Political Economy 1971; 79; 481-507.

Falk M, B Koebel, 1999, Curvature Conditions and Substitution Pattern among Capital, Energy, Materials and Heterogeneous Labour, no. no. 99-06, Mannheim, Mannheim.

Field BC, Grebenstein C. Capital-Energy Substitution in U.S. Manufacturing. Review of Economics and Statistics 1980; 62; 207-212.

Fuss MA. The Demand for Energy in Canadian Manufacturing. Journal of Econometrics 1977; 5; 89116.

Garofalo GA, Malholtra DM. Aggregation of Capital and Its Substitution with Energy. Eastern Economic Journal 1988; 14; 251-262. 
Goldstein H, Rasbash J. Efficient Computational Procedures for the Estimation of Parameters in Multilevel Models Based on Iterative Generalised Least Squares. Computational Statistics and Data Analysis 1992; 13; 63-71.

Gopalakrishnan C, Khaleghi GH, Shrestha RB. Energy Non-Energy Input Substitution in US Agriculture: Some Findings. Applied Economics 1989; 21; 673-679.

Greene WH. Econometric Analysis. Fourth Edition, Prentice Hall International, Inc.: Upper Saddle River, New Jersey; 2000.

Griffin JM. Engineering and Econometric Interpretations of Energy-Capital Complementarity: Comment. American Economic Review 1981; 71; 1100-1104.

Griffin JM, Gregory PR. An Intercountry Translog Model of Energy Substitution Responses. American Economic Review 1976; 66; 845-857.

Halvorsen R, Ford J 1979. Substitution among Energy, Capital, and Labor Inputs in U.S. Manufacturing. In: Pindyck RS (Eds), Advances in the Economics of Energy and Resources: The Structure of Energy Markets. JAI Press Inc.: Greenwich, Connecticut; 1979. p. 51-75.

Hanoch G. Production and Demand Models with Direct or Indirect Implicit Additivity. Econometrica $1975 ; 43 ; 395-419$.

Hedges LV 1994. Fixed Effects Models. In: Cooper H, Hedges LV (Eds), The Handbook of Research Synthesis. Russell Sage Foundation, New York; 1981. p. 285-299.

Hedges LV, Olkin I. Statistical Methods for Meta-Analysis. Academic Press: Orlando, Florida; 1985.

Hicks JR. The Theory of Wages. Macmillan: London; 1932.

Hisnanick JJ, Kyer BL. Assessing a Disaggregated Energy Input. Energy Economics 1995; 17; $125-$ 132.

Hisnanick JJ, Kymn KO. The Evaluation of the U.S. Manufacturing Sector Using Multifactor Inputs of Capital, Labor, Energy and Real Cash Balances. Rivista Internazionale di Scienze Economiche e Commerciali 1990; 37; 149-162.

Huang KS. Factor Demands in the U.S. Food-Manufacturing Industry. American Journal of Agricultural Economics 1991; 73; 615-620.

Ilmakunnas P, Törmä H. Structural Change in Factor Substitution in Finnish Manufacturing. Scandinavian Journal of Economics 1989; 91; 705-721.

Kemfert C. Estimated Substitution Elasticities of a Nested CES Production Function Approach for Germany. Energy Economics 1998; 20; 249-264.

Kemfert C, Welsch H. Energy-Capital-Labor Substitution and the Economic Effects of CO2 Abatement: Evidence for Germany. Journal of Policy Modeling 2000; 22; 641-660.

Khan AH. The Two-Level CES Production Function for the Manufacturing Sector of Pakistan. The Pakistan Development Review 1994; 28; 1-12.

Koetse MJ. Determinants of Investment Behaviour: Methods and Applications of Meta-Analysis. Dissertation Vrije Universiteit Amsterdam, Tinbergen Institute Research Series, no. 374, Amsterdam, The Netherlands; 2006.

Kuper GH, Soest DP van. Path-Dependency and Input Substitution: Implications for Energy Policy Modelling. Energy Economics 2002; 25; 397-407.

Morishima M. A Few Suggestions on the Theory of Elasticity (in Japanese). Keizai Hyoron (Economic Review) 1967; 16; 144-150.

Mulatu A. Relative Stringency of Environmental Regulation and International Competitiveness. Dissertation Free University, Tinbergen Institute Research Series, no. 332, Amsterdam, The Netherlands; 2004.

Nguyen SV, Streitwieser ML. Factor Substitution in U.S. Manufacturing: Does Plant Size Matter? Small Business Economics 1999; 12; 41-57.

Norsworthy JR, Harper MJ 1981. Dynamic Models of Energy Substitution in U.S. Manufacturing. In: Berndt ER, Field BC (Eds), Modeling and Measuring Natural Resource Substitution. MIT Press: Cambridge, Massachusetts; 1981. p. 177-208. 
Pindyck RS. Interfuel Substitution and the Industrial Demand for Energy: An International Comparison. Review of Economics and Statistics 1979; 61; 169-179.

Prywes M. A Nested CES Approach to Capital-Energy Substitution. Energy Economics 1986; 8; 22 28.

Rushdi AA. Economies of Scale and Factor Substitution in Electricity Supply Industry. Energy Economics $1991 ; 13 ; 219-229$.

Sutton AJ, Abrams KR, Sheldon TA, Song F. Methods for Meta-Analysis in Medical Research. John Wiley and Sons: New York; 2000.

Turnovsky MHL, Donnelly WA. Energy Substitution, Separability, and Technical Progress in the Australian Iron and Steel Industry. Journal of Business and Economic Statistics 1984; 2; 54-63.

Uzawa H. Production Functions with Constant Elasticities of Substitution. Review of Economic Studies 1962; 29; 291-299.

Walton AL. Variations in the Substitutability of Energy and Nonenergy Inputs: The Case of the Middle Atlantic Region. Journal of Regional Science 1981; 21; 411-420. 


\section{Appendix A: Estimating the standard errors of Morishima and cross-price elasticities}

Standard errors of effect sizes from primary studies are crucial ingredients in any meta-analysis. Unfortunately, only a few studies provide readily available standard errors, implying we have to calculate them by hand for most studies. For this purpose we use the Delta method (see Greene, 2000, pp. 357358). The derivation of a cross-price elasticity from a translog model estimation is given in Section 2. Applying the Delta method, the variance of this elasticity is given by:

$$
\operatorname{var}\left(C P E_{K E}\right)=\left(-1 / s_{K}\right)^{2} \times \operatorname{var}\left(\hat{\beta}_{K E}\right)
$$

which is straightforward to calculate when $\operatorname{var}\left(\hat{\beta}_{K E}\right)$ and $s_{K}$ are given in the primary study, as they usually are. Deriving the standard error of a $M E S$ elasticity is more complicated since we need information that is usually not given in the studies. Given the fact that the $M E S$ is equal to $C P E_{K E}-P E_{E}$, its variance is given by:

$$
\operatorname{var}\left(M E S_{K E}\right)=\operatorname{var}\left(C P E_{K E}\right)+\operatorname{var}\left(P E_{E}\right)-2 \times \operatorname{cov}\left(C P E_{K E}, P E_{E}\right)
$$

where $\operatorname{var}\left(C P E_{K E}\right)$ and $\operatorname{var}\left(P E_{E}\right)$ are the squared standard errors of $C P E_{K E}$ and $P E_{E}$, and the last term is the covariance between these two estimates. The latter is not given in primary studies and its calculation requires knowledge about the variance-covariance matrix. Therefore, we approximate the variance by (see Mulatu, 2004, p. 147):

$$
\operatorname{var}\left(M E S_{K E}\right)=\operatorname{var}\left(C P E_{K E}\right)+\operatorname{var}\left(P E_{E}\right)-2 r \times \operatorname{se}\left(C P E_{K E}\right) \operatorname{se}\left(P E_{E}\right)
$$

where $r$ is the correlation coefficient between $C P E_{K E}$ and $P E_{E}$. Of course, the correlation coefficient is not given in primary studies. However, a plausible assumption is that $r>0$ when $C P E_{K E}$ and $P E_{E}$ are of the same sign, and that $r<0$ when $C P E_{K E}$ and $P E_{E}$ have different signs. Furthermore, when we take a conservative value for $r$ we at least assure that the MES estimates for which we have to calculate standard errors ourselves will get conservative weights. Therefore, we set $r$ equal to 0.2 when $C P E_{K E}$ and $P E_{E}$ are of the same sign, and equal to -0.8 when $C P E_{K E}$ and $P E_{E}$ have different signs. These assumptions make it possible to estimate standard errors for most $M E S$ elasticities. 


\section{Appendix B: Characteristics of studies included in the meta-analysis}

Table B.1: Detailed characteristics of the translog studies included in the meta-analysis

\begin{tabular}{|c|c|c|c|c|c|c|c|c|}
\hline \multirow{2}{*}{ Study } & \multicolumn{2}{|c|}{ Number of estimates } & \multirow[t]{2}{*}{ M-incl } & \multirow{2}{*}{$\begin{array}{l}\text { Sample } \\
\text { size }\end{array}$} & \multirow[t]{2}{*}{ Data period } & \multirow{2}{*}{$\begin{array}{l}\text { Data } \\
\text { type }\end{array}$} & \multirow[t]{2}{*}{ RTS } & \multirow[t]{2}{*}{ NNTC } \\
\hline & $C P E_{K E}$ & $M E S_{K E}$ & & & & & & \\
\hline Berndt and Wood (1975) & 5 & 5 & Y & 25 & 1947-1971 & $\mathrm{T}$ & $\mathrm{N}$ & $\mathrm{N}$ \\
\hline Griffin and Gregory (1976) & 9 & 9 & $\mathrm{~N}$ & 36 & 1955-1969 & $\mathrm{P}$ & $\mathrm{N}$ & $\mathrm{N}$ \\
\hline Fuss (1977) & 1 & 1 & $\mathrm{Y}$ & 55 & $1961-1971$ & $\mathrm{P}$ & $\mathrm{Y}$ & $\mathrm{N}$ \\
\hline Berndt and Wood (1979) & 3 & 3 & $\mathrm{~N}$ & 25,55 & $1947-1971$ & $\mathrm{~T}$ & $\mathrm{~N}$ & $\mathrm{~N}$ \\
\hline Pindyck (1979) & 20 & 20 & $\mathrm{~N}$ & 110 & $1963-1973$ & $\mathrm{P}$ & $\mathrm{Y}$ & $\mathrm{N}$ \\
\hline Halvorsen and Ford (1979) & 28 & 28 & $\mathrm{~N}$ & Various & 1958 & $\mathrm{~T}$ & $\mathrm{~N}$ & $\mathrm{~N}$ \\
\hline Field and Grebenstein (1980) & 10 & 10 & $\mathrm{~N}$ & Various & 1971 & $\mathrm{C}$ & $\mathrm{N}$ & $\mathrm{N}$ \\
\hline Berndt and Wood (1981) & 6 & 6 & $\mathrm{~N}$ & Various & $\begin{array}{l}1947-1971 \\
1955-1969\end{array}$ & $\mathrm{~T}, \mathrm{P}$ & $\mathrm{N}$ & $\mathrm{N}$ \\
\hline Walton (1981) & 40 & 40 & Y & 24 & $1950-1973$ & $\mathrm{~T}$ & $\mathrm{~N}$ & $\mathrm{~N}$ \\
\hline Anderson (1981) & 3 & 3 & $\mathrm{Y}$ & 24 & $1948-1971$ & $\mathrm{~T}$ & $\mathrm{~N}$ & $\mathrm{~N}$ \\
\hline Norsworthy and Harper (1981) & 1 & 1 & Y & 20 & $1958-1977$ & $\mathrm{~T}$ & $\mathrm{~N}$ & $\mathrm{~N}$ \\
\hline Ball and Chambers (1982) & 0 & 1 & Y & 23 & $1954-1976$ & $\mathrm{~T}$ & Y & $\mathrm{Y}$ \\
\hline Dargay (1983) & 11 & 11 & $\mathrm{Y}$ & 25 & 1952-1976 & $\mathrm{T}$ & $\mathrm{N}$ & $\mathrm{N}$ \\
\hline Garofalo and Malhotra (1988) & 2 & 2 & $\mathrm{~N}$ & 360 & $1963-1978$ & $\mathrm{P}$ & $\mathrm{N}$ & Y \\
\hline Gopalakrishnan et al. (1989) & 1 & 1 & $\mathrm{~N}$ & 50 & 1982 & $\mathrm{C}$ & $\mathrm{N}$ & $\mathrm{N}$ \\
\hline Andrikopoulos et al. (1989) & 7 & 7 & $\mathrm{~N}$ & 21 & $1962-1982$ & $\mathrm{~T}$ & $\mathrm{Y}$ & $\mathrm{N}$ \\
\hline Debertin et al. (1990) & 0 & 1 & $\mathrm{Y}$ & 10 & 1970-1979 & $\mathrm{T}$ & $\mathrm{Y}$ & $\mathrm{Y}$ \\
\hline Hisnanick and Kymn (1990) & 6 & 6 & $\mathrm{~N}$ & 24 & 1958-1981 & $\mathrm{T}$ & $\mathrm{N}$ & $\mathrm{Y}$ \\
\hline Huang (1991) & 1 & 1 & $\mathrm{~N}$ & 16 & 1971-1986 & $\mathrm{T}$ & Y & Y \\
\hline Rushdi (1991) & 1 & 1 & $\mathrm{~N}$ & 35 & $1950-1984$ & $\mathrm{~T}$ & Y & $\mathrm{N}$ \\
\hline Hisnanick and Kyer (1995) & 2 & 2 & $\mathrm{Y}$ & 28 & $1958-1985$ & $\mathrm{~T}$ & $\mathrm{Y}$ & $\mathrm{N}$ \\
\hline Falk and Koebel (1999) & 1 & 0 & $\mathrm{Y}$ & 351 & $1978-1990$ & $\mathrm{P}$ & Y & Y \\
\hline
\end{tabular}

Number of estimates: Number of CPE and MES estimates provided by a study

M-incl:

Sample size:

Data period:

Data type:

RTS:

NNTC:
Model is estimated with materials as an explanatory variable; $\mathrm{Y}=\mathrm{Yes}, \mathrm{N}=\mathrm{No}$

Sample size used in the primary model estimations

Data period used in the primary model estimations

$\mathrm{C}=$ Cross-section data, $\mathrm{T}=$ Time series data, $\mathrm{P}=$ Panel data

Model is estimated with returns to scale parameters; $\mathrm{Y}=\mathrm{Yes}, \mathrm{N}=\mathrm{No}$

Model is estimated with non-neutral technological change parameters; $\mathrm{Y}=\mathrm{Yes}, \mathrm{N}=\mathrm{No}$ 